\title{
Percutaneous transfemoral transcatheter mitral valve replacement of a degenerated mitral bioprosthesis: initial experience in Southeast Asia with nine-month follow-up \\ Singapore Med J 2020; 61(8): 445-446 https://doi.org/10.11622/smedj.2020019
}

Dear Sir,

Transcatheter aortic valve implantation (TAVI) or transcatheter aortic valve replacement (TAVR) has become a treatment option for patients with severe aortic valve stenosis who are inoperable or at high and intermediate surgical risk. ${ }^{(1)}$ Transcatheter aortic valve-invalve replacement for degenerated surgical aortic bioprostheses has also become an established, less invasive alternative to redo open aortic valve replacement. ${ }^{(2)}$ Transcatheter mitral valve-in-valve replacement has been employed in smaller numbers for degenerated mitral bioprostheses. ${ }^{(3)}$ To our knowledge, our case is the initial experience in Southeast Asia of percutaneous transfemoral transcatheter mitral valve-in-valve replacement for a failed surgical mitral bioprosthesis, with nine months' follow-up.

A 64-year-old obese man was referred to our institution for exertional dyspnoea (NYHA [New York Heart Association] Class III) and orthopnoea of 3-4 months' duration. His past medical history included chronic renal failure (serum creatinine $\sim 400 \mu \mathrm{mol} / \mathrm{L}$ ), diabetes mellitus, hypertension, dyslipidaemia and rheumatic heart disease with mitral valve replacement (27-mm Carpentier-Edwards PERIMOUNT Magna bioprosthesis [Edwards Lifesciences, Irvine, CA, USA]) 9.5 years prior to the current presentation.

Echocardiography showed very severe mitral stenosis (MS) due to a degenerated mitral bioprosthesis with a mean pressure gradient (MPG) of $22 \mathrm{mmHg}$ (vs. baseline mitral MPG nine years ago of $8 \mathrm{mmHg}$ ) and valve area of $0.8 \mathrm{~cm}^{2}$ (Box 1). There was severe pulmonary hypertension with pulmonary artery systolic pressure (PASP) of $81 \mathrm{mmHg}$. Left ventricular ejection fraction was $60 \%$. Due to the patient's elevated surgical risk (obesity [body mass index $30 \mathrm{~kg} / \mathrm{m}^{2}$, body surface area $1.9 \mathrm{~m}^{2}$ ], severe pulmonary hypertension, chronic renal failure and redo open heart surgery), he was referred for percutaneous transcatheter mitral valve-in-valve replacement.

The procedure was performed under general anaesthesia with transoesophageal echocardiography and fluoroscopic imaging. A pacing wire was advanced via the left femoral vein to the right ventricular apex. A transseptal puncture was performed via the right femoral vein to gain access to the left atrium (Fig. 1). A balloon-expandable 29-mm Edwards SAPIEN 3 valve (Edwards Lifesciences, Irvine, CA, USA) was positioned within the failed mitral bioprosthesis (Fig. 2) and deployed under rapid ventricular pacing, achieving a good position (Fig. 3). Recovery was uneventful and the patient was discharged two days after the procedure. Echocardiography showed a stable SAPIEN 3 valve within the Magna bioprosthesis, a mitral MPG of $10 \mathrm{mmHg}$, trivial mitral regurgitation and a significant decrease in pulmonary pressure (PASP $51 \mathrm{mmHg}$ ). The patient was well at the 30-day and nine-month follow-up and reported improved functional status (NYHA Class I-II) and resolution of his orthopnoea. Serum creatinine remained stable and echocardiography revealed satisfactory valve haemodynamics (mitral valve MPG $11 \mathrm{mmHg}$, mitral valve area $\sim 1.4 \mathrm{~cm}^{2}$, PASP $55 \mathrm{mmHg}$ ).

TAVI has become an established therapeutic option for patients with severe aortic valve stenosis, ${ }^{(1)}$ and transcatheter aortic valve-in-valve replacement is increasingly accepted as a less invasive and lower-risk treatment for patients with degenerated aortic bioprostheses. $^{(2,4)}$ Similarly, transcatheter mitral valve-in-valve replacement, although currently an off-label indication, is an attractive alternative to redo open heart surgery for many patients with degenerated mitral bioprosthesis, especially for high-risk patients such as our case.

Percutaneous transcatheter mitral valve-in-valve replacement for failed mitral bioprosthesis is, however, less commonly performed, with the majority of cases using the direct transapical approach. ${ }^{(3)}$ A transpical approach was the route of choice in our early Singapore

\footnotetext{
Box 1. 2014 American College of Cardiology/American Heart Association classification of native mitral stenosis (MS): Progressive MS

- Mitral valve area (by planimetry) $>1.5 \mathrm{~cm}^{2}$

- Transmitral mean pressure gradient usually $<5 \mathrm{mmHg}$ Severe MS

- Mitral valve area (by planimetry) $<1.5 \mathrm{~cm}^{2}$

- Transmitral mean pressure gradient usually $>5 \mathrm{mmHg}$ to $10 \mathrm{mmHg}$

Very severe MS

- Mitral valve area (by planimetry) $<1.0 \mathrm{~cm}^{2}$

- Transmitral mean pressure gradient usually $>5 \mathrm{mmHg}$ to $10 \mathrm{mmHg}$

- Mitral valvuloplasty or mitral valve replacement is indicated for symptomatic patients with severe or very severe MS. In patients with progressive rheumatic MS and symptoms, mitral valvuloplasty can be considered.
}

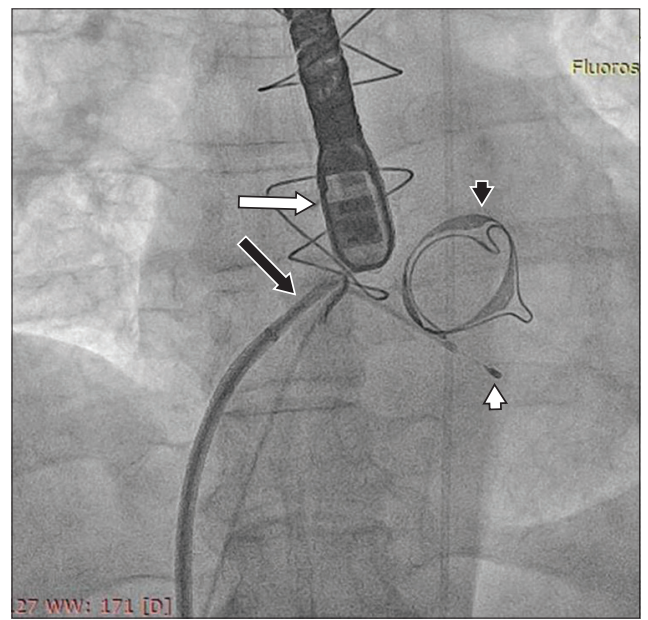

Fig. 1 Fluoroscopic image shows the TEE probe (white arrow), the transseptal sheath and needle (black arrow), the pacing wire in the right ventricular apex (white arrowhead), and the degenerated Carpentier-Edwards PERIMOUNT Magna valve (black arrowhead). TEE: transoesophageal echocardiography 


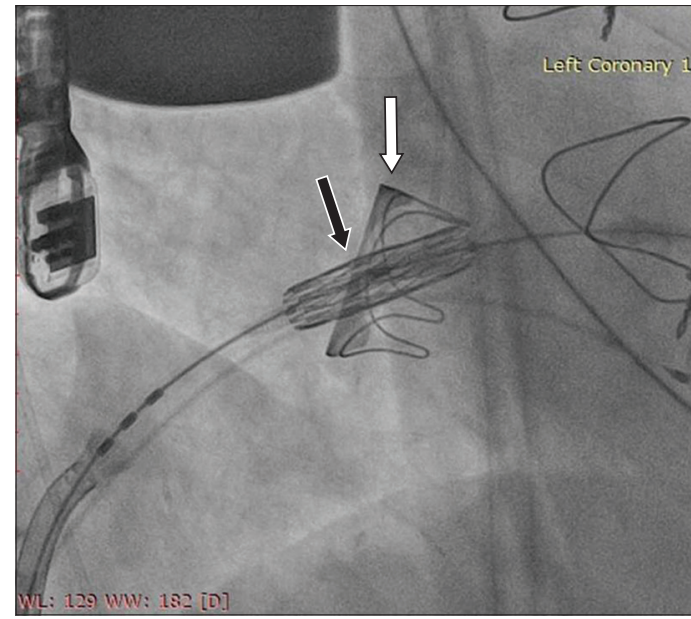

Fig. 2 Fluoroscopic image shows the Edwards SAPIEN 3 transcatheter heart valve (black arrow) within the Carpentier-Edwards PERIMOUNT Magna valve (white arrow).

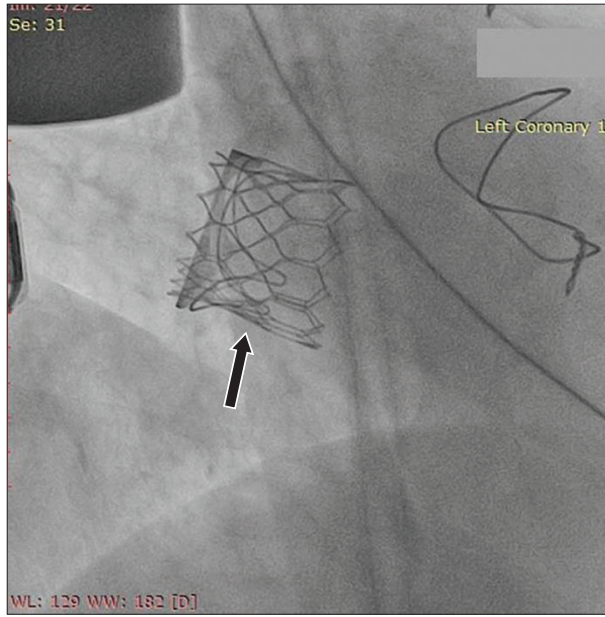

Fig. 3 Fluoroscopic image shows the fully deployed Edwards SAPIEN 3 transcatheter heart valve (arrow) within the failed mitral bioprosthesis.

experience with mitral valve-in-valve replacement due to the proximity of the left ventricular apex to the mitral valve and the coaxial delivery of the transcatheter heart valve. ${ }^{(5)}$ However, it requires a surgical lateral thoracotomy, leading to a longer recovery period with the risk of left ventricular injury, atrial fibrillation, pleural effusion and cardiac apex bleeding. ${ }^{(6,7)}$

A true percutaneous technique using a transvenous (femoral vein) approach is inherently more attractive due to its less invasive nature, reduced bleeding risk and shorter hospital stay. Nevertheless, it is more challenging, requiring transseptal puncture skills and greater wire and catheter manipulations. Several studies have shown a procedural success rate of between $82 \%$ and $94 \%$ as well as satisfactory short-term outcomes. ${ }^{(3,8,9)}$

We herein report the initial experience of a transvenous transseptal mitral valve-in-valve replacement in Southeast Asia using the Edwards SAPIEN 3 balloon-expandable valve. An oversized SAPIEN 3 transcatheter valve was selected because the transcatheter valve must be larger than the previous bioprosthesis to anchor within it (the internal diameter of the 27-mm Carpentier-Edwards PERIMOUNT Magna valve was $26 \mathrm{~mm}$ ). An appropriately sized transcatheter heart valve also optimises haemodynamics and minimises paravalvular leaks.

This case demonstrates that the percutaneous transfemoral (transvenous) route for transcatheter mitral valve-in-valve replacement is feasible, leading to a successful procedure, a short hospital stay, preservation of renal function and a good clinical outcome at nine months. Mitral valvuloplasty or mitral valve replacement is indicated for symptomatic patients with severe or very severe MS. In patients with progressive MS and symptoms without other cause, mitral valvuloplasty can be considered.

Yours sincerely,

Paul Toon Lim $\underline{\text { Chiam }}^{1,2}$, Yean Teng $\underline{\underline{\operatorname{Lim}}}{ }^{1,2}$, Choon Pin $\underline{\operatorname{Lim}}^{1}$, Cumaraswamy $\underline{\text { Sivathasan }}^{1}$

${ }^{1}$ Mount Elizabeth Hospital, ${ }^{2}$ National University of Singapore, Singapore. paulchiam@heartvascularcentre.com

\section{References}

1. Chiam PTL. Transcatheter aortic valve implantation in Asia: the first decade. Eurolntervention 2018; 14:35-7.

2. Dvir D, Webb JG, Bleiziffer S, et al; Valve-in-Valve International Data Registry Investigators. Transcatheter aortic valve implantation in failed bioprosthetic surgical valves. JAMA 2014; 312:162-70.

3. Yoon SH, Whisenant BK, Bleiziffer S, et al. Outcomes of transcatheter mitral valve replacement for degenerated bioprostheses, failed annuloplasty rings, and mitral annular calcification. Eur Heart J 2019; 40:441-51.

4. Chiam PT, Ewe SH, Soon JL, et al. Percutaneous transcatheter aortic valve implantation for degenerated surgical bioprostheses: the first case series in Asia with oneyear follow-up. Singapore Med J 2016; 57:401-5.

5. Soon JL, Chua YL, Chao VT, et al. Asia's first successful minimally invasive transapical transcatheter mitral valve-in-valve implantation. Ann Acad Med Singapore $2013 ; 42: 85-7$.

6. Seiffert M, Conradi L, Baldus S, et al. Transcatheter mitral valve-in-valve implantation in patients with degenerated bioprostheses. JACC Cardiovasc Interv 2012; $5: 341-9$.

7. Dvir D. Transseptal instead of transapical valve implantation: making mitral great again? JACC Cardiovasc Interv 2016; 9:1175-7.

8. Bouleti C, Fassa AA, Himbert D, et al. Transfemoral implantation of transcatheter heart valves after deterioration of mitral bioprosthesis or previous ring annuloplasty. JACC Cardiovasc Interv 2015; 8(1 Pt A):83-91.

9. Eleid MF, Cabalka AK, Williams MR, et al. Percutaneous transvenous transseptal transcatheter valve implantation in failed bioprosthetic mitral valves, ring annuloplasty, and severe mitral annular calcification. JACC Cardiovasc Interv 2016; 9:1161-74. 